\title{
Functional Retroperitoneal Neuroblastoma and Ganglioneuroblastoma in Adults
}

\author{
${ }^{1}$ Kamilla Mahkamova, ${ }^{2}$ Barney J Harrison
}

\section{ABSTRACT}

Ganglioneuroblastoma and neuroblastoma are malignant catecholamine-secreting tumors arising from neural crest tissue. Thirty-eight cases of functional retroperitoneal extra-adrenal neuroblastoma and ganglioneuroblastoma in adults are reported in English literature. These tumors behave more aggressively in adults compared to children and have very poor prognosis. We report four adults who presented with retroperitoneal extraadrenal ganglioneuroblastoma and neuroblastoma between 2008 and 2012. Median age at presentation was 28.5 years (21-40 years). One patient was in late stages of pregnancy. Three patients presented with local pressure effects from the tumor and one patients manifested clinical features of catecholamine excess. Biochemical analysis showed raised urinary dopamine excretion in all our patients. Urinary noradrenaline and metanephrines were elevated in three cases. Computed tomography/magnetic resonance imaging/meta-iodobenzylguanidine (CT/MRI/MIBG) confirmed stage IIB disease in one patient, stage III disease one patient and stage IV in two cases. Preoperative biopsy confirmed diagnosis in two cases. Complete surgical resection was performed in patient with stage II B disease and partial surgical resection was feasible in other cases. Histological diagnosis was ganglioneuroblastoma in two cases and neuroblastoma in two cases. Meta-iodobenzylglunidine/radiotherapy and chemotherapy were used for residual disease. Urinary catecholamines and metanephrines normalized after complete tumor resection but remained elevated in residual disease. Survival for neuroblastoma was 15 and 21 months after the surgery and 29 months for ganglioneuroblastoma. One patient remains free of disease at 6 months. Functioning ganglioneuroblastoma and neuroblastoma are rare in adults and only one other case ganglioneuroblastoma in pregnancy is reported in the world literature. Measurement of plasma/urinary excretion of catecholamines and their precursors and metabolites is helpful for diagnosis of neuroblastoma and ganglioneuroblastoma. Due to late presentation surgical resection is unlikely to be curative. Three of our patients developed local recurrence of disease. Urinary/plasma assay are used for monitoring disease relapse. Long-term survival is unusual.

Keywords: Neuroblastoma, Ganglioneuroblastoma, Catecholamine-secreting tumors, Extra-adrenal tumors, Adults.

${ }^{1}$ Trainee (Core Surgery), ${ }^{2}$ Consultant (General Surgeon)

${ }^{1}$ Department of General Surgery, Chesterfield Royal Hospital NHS Foundation Trust, Chesterfield, Derbyshire, United Kingdom

${ }^{2}$ Department of Endocrine Surgery, Royal Hallamshire Hospital, Sheffield, South Yorkshire, United Kingdom

Corresponding Author: Kamilla Mahkamova, Trainee (Core Surgery), Department of General Surgery, Chesterfield Royal Hospital, NHS Foundation Trust, Chesterfield, Derbyshire, Glossop Road, United Kingdom, e-mail: kamilla65@hotmail.com
How to cite this article: Mahkamova K, Harrison BJ. Functional Retroperitoneal Neuroblastoma and Ganglioneuroblastoma in Adults. World J Endoc Surg 2014;6(2):89-95.

\section{Source of support: Nil}

\section{Conflict of interest: None}

\section{INTRODUCTION}

Neuroblastoma and ganglioneuroblastoma are malignant tumors that arise from primitive neural crest cells. The incidence of neuroblastoma is approximated as 2.26 per million person-years, with $60 \%$ of cases diagnosed before the age of 2 years and $84 \%$ before the age of 10 years. It is extremely rare in adults (around 0.1-0.2 cases per million per year in the United States). ${ }^{1,2}$ Ganglioneuroblastoma in adults is even less prevalent: there are only fifty cases of adult ganglioneuroblastoma reported in the literature. ${ }^{3}$ In adults, common primary sites of neuroblastoma are pelvis, abdomen and retroperitoneum. ${ }^{4,5}$ Ganglioneuroblastoma tend to occur in the abdomen, mediastinum, neck and lower extremities. ${ }^{6}$

Catecholamine overproduction associated with neuroblastoma was first described in 1959 by Issacs et al. ${ }^{7}$ Further observations by Sandler in 1967 suggest that $75 \%$ of neuroblastoma/ganglioneuroblastoma are functional, they invariably secrete dopamine. ${ }^{8}$ Symptoms of catecholamine production, however, do not commonly feature in the presentation of these tumors and the main presenting symptoms are due to local pressure effects from the mass or from disseminated disease.

Neuroblastoma tend to be more aggressive than ganglioneuroblastoma, both metastasise through lymphatic and vascular routes, to bone, liver, skin and genitalia. Paucity of information is available on the tumor behavior in adults and no standard guidelines for managing adult neuroblastoma and ganglioneuroblastoma are available at present.

We describe four patients (two males and two females, median age 28.5 years - range 21-40 years) referred with a retroperitoneal mass subsequently diagnosed as catecholamine producing retroperitoneal neuroblastoma or ganglioneuroblastoma. One of the patients presented with ganglioneuroblastoma in the late stages of pregnancy, only the second case reported in the world literature. This paper aims to raise the awareness of functional retroperitoneal 
(extra-adrenal) neuroblastoma and ganglioneuroblastoma occurring in adulthood and reports the tumors' distinct biochemical and pathological features, which help to differentiate them from other adult malignancies that can present in similar manner, including primary or metastatic small cell carcinoma, lymphoma, rhabdomyosarcoma and paraganglioma. The current treatment strategies and outcome are also discussed.

\section{CASE REPORTS}

\section{Case 1}

A 36-year-old male with a 3-year history of severe back pain was found on computed tomography $(\mathrm{CT})$ to have a retroperitoneal mass measuring $10 \times 9 \mathrm{~cm}$ and skeletal changes suspicious of metastases. Bone marrow biopsy stained positive for CD56 and showed malignant cells with neuroendocrine differentiation. Urinary noradrenaline, normetanephrine and dopamine excretion was raised at $0.8,34.4$ and $55.2 \mu \mathrm{mol} /$ 24 hour, respectively (normal ranges: noradrenaline: 0-0.49 $\mu \mathrm{mol} / 24 \mathrm{hrs}$, normetanephrines $0-3.0 \mu \mathrm{mol} / 24 \mathrm{hrs}$ dopamine $0-3.4 \mu \mathrm{mol} / 24 \mathrm{hrs}$ ). The initial diagnosis was of malignant abdominal paraganglioma.

Perioperative $\alpha$-adrenoceptor blockade was achieved with phenoxybenzamine. At laparotomy a tumor invading the posterior abdominal wall and encircling the aorta and vena cava was partially excised leaving residual disease on the vertebral column. Macroscopic analysis reported a $100 \times$ $90 \times 65 \mathrm{~mm}$ tumor; the cut surface was brown with bright yellow patches. Microscopy showed a malignant tumor with evidence of widespread vascular invasion, and birefringent yellow crystals, presumed to be rich in tyrosine. Cells were negative for v-myc avian myelocytomatosis viral oncogene neuroblastoma (MYCN) amplification, moderately positive for CD56 and synaptophysin, strongly positive for neurofilament and focally positive for S-100 and microtubule associated protein (MAP-2) in larger cells. The findings were consistent with pure adult type neuroblastoma, owing to the absence of characteristic cell groupings found with paraganglioma.

At 2 months after surgery, meta-iodobenzylguanidine (MIBG) scan confirmed widespread bone metastases. Post-operative urinary excretion of noradrenaline, normetanephrine and dopamine remained elevated at 0.9, 45.6 and $241.4 \mu \mathrm{mol} / 24$ hours, respectively.

Therapeutic MIBG was administered and following three treatment sessions the patient reported improvement in his symptoms, which were mainly lower back pain.

Several months later, he developed further metastases in his thoracic spine and skull and required further surgery to decompress thoracic vertebrae and resect skull metastasis.
Localized radiotherapy to the thoracic spine was administered postoperatively.

Disease progression was demonstrated in the liver and skeleton and following palliative chemotherapy (Etopside), the patient succumbed to the disease 21 months after the initial surgery.

\section{Case 2}

A 40-year-old woman complaining of left loin pain was found on CT scan to have a $70 \times 40 \mathrm{~mm}$ retroperitoneal mass encasing the left renal artery and evidence of retroperitoneal lymphadenopathy. The mass was biopsied under CT guidance which confirmed the presence of a tumor with neuroendocrine differentiation. A 24-hour urine collection revealed normal levels of adrenaline, noradrenaline, metanephrine and normetanephrine. Urinary dopamine was grossly elevated on more than one occasion at 19.9, 20.9 and $13.5 \mu \mathrm{mol} / 24$ hours $(3.40-3.85 \mu \mathrm{mol} / 24 \mathrm{hrs})$. Metaiodobenzylguanidine scan showed vague uptake in the left acetabulum, suggestive of metastasis.

Left nephrectomy, adrenalectomy and splenectomy were performed; histology reported a poorly differentiated ganglioneuroblastoma of abdominal autonomic ganglion origin. Focal Homer-Wright rosettes were observed and small to medium cells with scant cytoplasm were seen. The tissue was negative for MYCN amplification. Clusters of cells stained positive for $\mathrm{S} 100$, neurofilament, microtubule associated protein-2 (MAP-2), CD56, neuron-specific enolase, prostatic acid phosphatase 9.5 and synaptophysin.

Postoperative magnetic resonance imaging (MRI) identified a left ovarian mass; this was excised and confirmed as a metastatic tumor of neuroectodermal origin.

The patient developed local recurrence at the site of initial surgery, MIBG scan confirmed metastatic disease in the spine, liver, mid upper abdomen and pelvis. The patient was offered chemotherapy but declined this in favor of therapeutic MIBG. Her symptoms of back pain were alleviated following therapeutic MIBG, but soon after completing the treatment, she developed anemia. Bone marrow biopsy showed infiltration by tumor cells but the patient declined further treatment. She died 29 months after the initial surgery.

\section{Case 3}

A 21-year-old man presented with a 1 year history of progressively worsening low back pain, constipation not relieved by laxatives and weight loss. This was associated with episodes of palpitations, excessive sweating and headaches. He had no significant family history.

Clinical examination was unremarkable apart from moderately elevated blood pressure (BP 157/77 mm Hg). 
Computed tomography of abdomen and pelvis (Fig. 1), identified a large retroperitoneal mass extending from the superior border of right kidney to the aortic bifurcation, crossing the midline and associated with enlarged retroperitoneal lymph nodes.

Core biopsy of the mass confirmed the mass to be a grade II neuroendocrine tumour - a poorly differentiated neuroblastoma.

A 24 hour urine collection showed significantly raised dopamine and high normetanephrine levels. Plasma normetanephrine levels were elevated (Table 1).

Meta-iodobenzylguanidine and bone marrow biopsy ruled out distant metastatic disease.

The patient received neoadjuvant chemotherapy, which resulted in a slight reduction of the size of the retroperitoneal mass. Laparotomy was performed after preoperative alpha-blockade. Intraoperative findings included a tumor mass extending from the level of the right kidney deep into the pelvis with the tumor bulk overlying and encasing the abdominal aorta and inferior vena cava, renal vessels and right ureter. Infra renal resection of the tumor was performed (approximately $50 \%$ of the tumor mass was removed).

Histology confirmed the ultrastructural features of neuroblastoma, i.e. cluster of small round chromatin-rich cells in a fibrocellular tissue stroma with positive immunohistochemical staining for MAP-2 and neurofilament. Evidence of extensive vascular invasion was demonstrated.

The postoperative recovery was complicated by the development of recurrent retroperitoneal fluid collections managed with percutaneous drainage under CT guidance and antibiotic therapy.

Postoperatively, the patient underwent further chemotherapy but a progressive increase in the size of the tumor was observed on subsequent CT. The patient died from disease 6 months after the initial surgery.

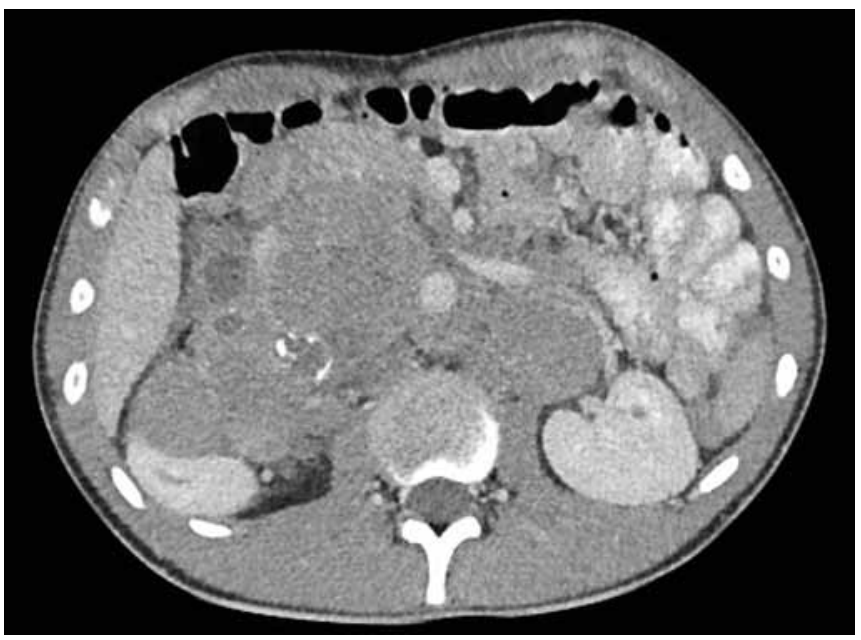

Fig. 1: Abdominal CT of Patient 3 showing large retroperitoneal mass which crossed the midline
Table 1: Biochemical profile for Patient 3

\begin{tabular}{lll}
\hline & Values & $\begin{array}{l}\text { Reference } \\
\text { ranges }\end{array}$ \\
\hline 24-hour urine dopamine & $7392 \mathrm{nmol} / 24 \mathrm{hrs}$ & $0-3400$ \\
24-hour urine methoxytyramine & $5.2 \mu \mathrm{mol} / 24 \mathrm{hrs}$ & $2.4-2.8$ \\
24-hour urinary metanephrine & $0.8 \mu \mathrm{mol} / 24 \mathrm{hrs}$ & $0-1.7$ \\
24-hour urinary normetanephrine & $8.8 \mu \mathrm{mol} / 24 \mathrm{hrs}$ & $0-3.0$ \\
24-hour urine noradrenaline & $357 \mu \mathrm{mol} / 24 \mathrm{hrs}$ & $0-490$ \\
Plasma metanephrine & $187 \mathrm{pmol} / \mathrm{l}$ & $80-510$ \\
Plasma normetanephrine & $2025 \mathrm{pmol} / \mathrm{l}$ & $120-1180$ \\
\hline
\end{tabular}

Table 2: Biochemical profile for Patient 4

\begin{tabular}{lll}
\hline & Values & $\begin{array}{l}\text { Reference } \\
\text { ranges }\end{array}$ \\
\hline 24-hour urine dopamine & $\begin{array}{l}4929 \mathrm{nmol} / 24 \mathrm{hrs} \\
(1 \mathrm{st} \mathrm{sample})\end{array}$ & $0-3400$ \\
& $\begin{array}{l}22746 \mathrm{nmol} / 24 \mathrm{hrs} \\
(2 \mathrm{nd} \mathrm{sample})\end{array}$ & \\
& 11365 & $0-2300$ \\
$\begin{array}{l}\text { 24-hour urine 3- } \\
\text { methoxytyramine }\end{array}$ & 612 & $0-1000$ \\
24-hour urine metanephrine & $0-3000$ \\
\hline
\end{tabular}

\section{Case 4}

A 23-year-old primigravida, 33 week pregnant, was referred with an incidental finding of a large retroperitoneal mass identified on abdominal ultrasound scan after a fall. Past medical and family histories were noncontributory. Physical examination revealed a distended abdomen consistent with gravid uterus and no other palpable masses. The patient was normotensive.

Urinary dopamine levels were elevated on two occasions and urinary normetanephrine levels were found to be above the normal limit (Table 2).

Magnetic resonance imaging scan demonstrated a large homogeneous soft tissue mass in the right retroperitoneal space extending paravertebrally from the level of L2 to T9 (Fig. 2). The mass displaced the right kidney and

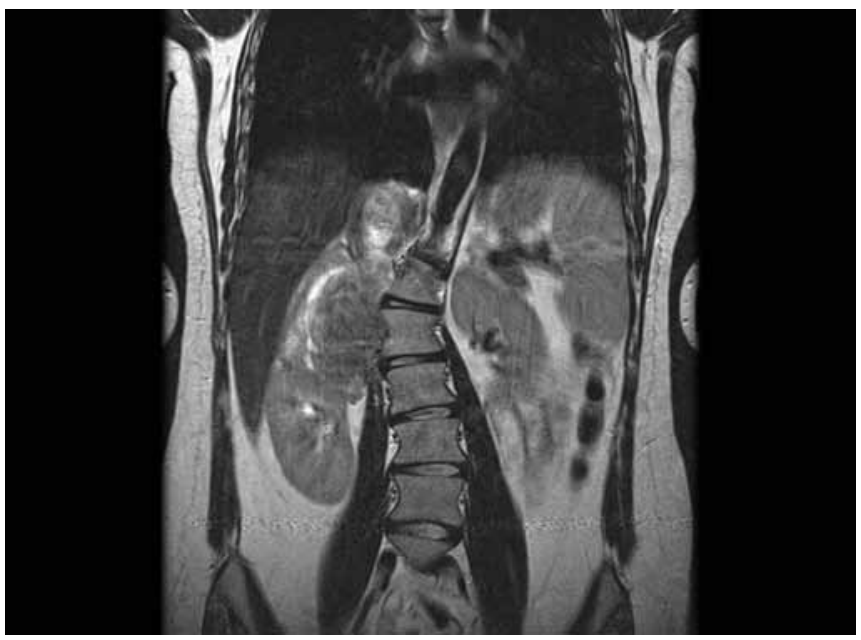

Fig. 2: Magnetic resonance imaging abdomen image of Patient 3 showing tumor mass in right retroperitoneal space extending into thorax 
invaded the thorax through defect in the posterior aspect of diaphragm.

As malignancy was suspected, the patient underwent cesarean delivery at 36 weeks gestation after preoperative administration of $\alpha$-blockade. A live male infant was delivered without significant complications.

After delivery, FDG PET whole body scan showed 'low levels of activity in the tumor suggestive of non-aggressive disease'. The patient underwent curative resection of the lesion via a transthoracic approach 3 weeks after the cesarean section. Intraoperative findings were of a lobulated tumor mass, the main bulk situated behind the right kidney and medially behind the inferior vena cava. A separate tumor nodule was excised from the thoracic aspect of the diaphragm.

The tumor $(17 \times 13 \times 5 \mathrm{~cm}, 540 \mathrm{~g}$ with the diaphragmatic nodule measuring $8 \times 5 \times 3 \mathrm{~mm}$ ) consisted of primitive neuroblasts and ganglion cells, i.e. ganglioneuroblastoma of origin from the sympathetic chain.

Postoperatively, plasma dopamine levels were within the normal range. The patient is under life-long follow-up and monitored with 6 monthly plasma normetanephrines and dopamine and 6 monthly MRI scans.

\section{DISCUSSION}

Literature search for adult (over 16 years of age) retroperitoneal (extra-adrenal) neuroblastoma and ganglioneuroma using Medline, Embase and PubMed identified 26 cases of histologically diagnosed retroperitoneal neuroblastoma (including one of renal origin) $)^{4,5,9-24}$ and 12 cases of ganglioneuroblastoma (including 2 renal and 1 paravertebral in origin). ${ }^{625-32}$ In the neuroblastoma group, the median age was 32 (16.1-56). There were 9 males and 17 females. In the ganglioneuroblastoma group, there were 6 males and 6 females with median age 46.5 (26-69).

From the literature review it is difficult to infer the proportion of catecholamine-producing retroperitoneal neuroblastoma and ganglioneuroblastoma and the nature of the secreted bioamines due to insufficient data. In 8 of 26 cases of adult retroperitoneal neuroblastoma, 24-hour urine excretion of catecholamines and their metabolites were measured. The levels were found to be elevated in five of these cases. ${ }^{9,11,12,15,17}$ Vanillylmandelic acid (VMA) and urinary metanephrine levels were most commonly specified as being raised.

Assay of urinary catecholamines and their metabolites was performed in 5 out of total of 12 cases of ganglioneuroblastoma. The levels were elevated in 2 out of 5 patients. ${ }^{6,27}$ Raised VMA levels were found in one of the cases, and in the second case no further details as to the nature of raised urinary catecholamines were provided.
All of the patients in our case series demonstrated raised urinary dopamine excretion. Patient 2 had an exclusively dopamine secreting ganglioneuroblastoma, whereas Patient 1 and Patient 3 presented with noradrenaline-producing neuroblastoma.

In previous studies involving 144 children $^{7,33-40}$ diagnosed with neuroblastoma or ganglioneuroblastoma at different anatomical sites, the proportion of catecholamine secreting tumors varied between 75 and 100\% depending on the case series, they were invariably dopamine secretors. Elevated urinary excretion of noradrenaline/total metanephrines/VMA was described in at least $65 \%$ of cases. A case of adrenaline secreting ganglioneuroblastoma is reported. ${ }^{41}$

This pattern of urinary bioamine excretion may suggest a diagnosis of paraganglioma/pheochromocytoma and this was the initial diagnosis for Patient 1 and Patient 3. In addition, dopamine secretion by pheochromocytoma is rare and often an indication of malignancy. ${ }^{42}$

One of our patients, Patient 3 with a noradrenaline- producing neuroblastoma had clinical features of catecholamine excess, which is more indicative of pheochromocytoma. Noradrenaline-secreting neuroblastoma produce hypertension in a minority of cases. ${ }^{43}$ To explain this, Sadler and Ruthven ${ }^{8,44}$ proposed that noradrenaline is metabolically degraded in tumor tissue or peripherally and only tiny quantities of free noradrenaline are liberated into the circulation. It would however seem prudent that when biopsy or surgical resection is to be performed in patients with noradrenaline producing tumors, pre-procedure $\alpha$-adrenoceptor blockade is used as a means to avoid hypertensive crisis. ${ }^{45}$

Histological diagnosis of neuroblastoma and ganglioneuroblastoma is confirmed by the typical appearance of small round cells with hyperchromatic nuclei and scanty cytoplasm, divided by fibrovascular stroma, neuroblastomas often exhibit typical Homer-Wright rosettes formations). Abortive pseudorosettes may be seen in undifferentiated neuroblastoma.

Negative immunohistochemical staining for leukocyte antigen (CD45), muscle markers and cytokeratins and positive neural markers CD 56, chromogranin A, synaptophysis, neurophilament and neuron specific enolase help to differentiate neuroblastomas from other tumors of non-neuronal origin. ${ }^{20}$

Meta-iodobenzylguanidine is sensitive in detecting distant soft tissue and bony metastasis of neuroblastoma. Metaanalysis of seven pediatric study series reported sensitivity greater than $76 \%$ (76-100\%). Meta-iodobenzylguanidine is recommended for the initial staging of the disease and for reassessment following treatment. ${ }^{46}$

The treatment protocols for adult neuroblastoma and ganglioneuroblastoma are adapted from pediatric guidelines 
and guided mainly by disease stage and histological features of the tumor. Staging is in accordance with the International Neuroblastoma Staging System (Table 3). ${ }^{47}$

Adults with retroperitoneal neuroblastoma/ganglioneuroblastoma tend to present with advanced stage disease and metastasis to multiple different sites more frequently than pediatric cases. ${ }^{13}$ Of 26 patients with retroperitoneal neuroblastoma one patient had stage I disease, one patient had stage II disease, 8 had stage III disease and 16 had stage IV disease. In 12 cases of ganglioneuroblastoma, 4 were known to have metastatic spread. Even localized disease appears to follow a more aggressive course in adults compared with children. ${ }^{5,13,19,25,48}$

Patients with localized disease (stage I and II) are treated with surgical resection of the tumor. Survival data are only available from pediatric studies reporting 90\% 3-year eventfree survival. ${ }^{49,47}$

Recurrent or disseminated disease (Stage III and IV) warrants treatment with chemotherapy and/or radiotherapy. Surgery still has a role in advanced disease, aggressive surgical intervention in children as initial treatment is reported to have a favorable effect on the prognosis. ${ }^{4}$

Combination chemotherapy with multiple cytotoxic agents can achieve significant regression of the tumour in children, ${ }^{50,51}$ but in adults the results are more disappointing. Although, chemotherapy rendered beneficial responses in some adult patients' numbers, there is no significant impact on overall survival. ${ }^{4,19,22,25,52}$

In selected patients with recurrent or refractory disease, I131MIBG therapy may be appropriate. An on-going clinical trial reports overall (complete and partial) response rates around $36 \%$ and significant increase in overall survival for neuroblastoma patients ( $49 \%$ at 1 year and $29 \%$ at 2 years). The significant complication of therapeutic MIBG therapy is hematologic toxicity. Myelosuppression was observed at higher doses (15 mCi/kg or higher), with $50 \%$ of subjects requiring hematopoietic cell transfusion. ${ }^{53}$

Scarce data are available to draw any conclusions on the efficacy of radiotherapy in adults with neuroblastoma and ganglioneuroblastoma.

Urinary or plasma assay of catecholamines and their metabolites are used to monitor response to the therapy and for relapsed disease. Dopamine appears to be a more reliable indicator of persistent disease/relapse.

A more favorable prognosis is associated with younger age, localized disease (stage I and II) at presentation and the type of tumor. Patients with ganglioneuroblastoma have a better prognosis compared to those with neuroblastoma.

The average survival for our patients with neuroblastoma was 13.5 months after the initial surgery and 29 months for ganglioneuroblastoma with one patient with ganglioneuroblastoma remains free of disease following surgery.

From the literature review, 21 out of 26 cases $(80.8 \%)$ of adult retroperitoneal neuroblastoma had follow-up of more than 12 months. At 12 months, 15 out of 21 (71.4\%) patients were alive with or without disease. For adult retroperitoneal ganglioneuroblastoma, data for 12 months survival was available for 8 patients $(66.7 \%)$. Four patients remained alive at 1 year $(50 \%)$, though one of the deaths was due to suicide rather then the disease.

The survival rates at 2 and 3 years cannot be accurately estimated from the literature review due to insufficient follow-up. Five patients with neuroblastoma presented with recurrent/metastatic disease within 2 to 3 years. The overall survival is very poor.

\section{LEARNING POINTS}

- Neuroblastoma and ganglioneuroblastoma are a very rare cause of retroperitoneal tumor in adults but should be considered in the differential diagnosis.

Table 3: International neuroblastoma staging system

\begin{tabular}{|c|c|}
\hline Stages & Disease involvement \\
\hline Stage I & $\begin{array}{l}\text { Localized tumor with complete gross excision, with or without microscopic residual disease; representative ipsilateral lymph } \\
\text { nodes negative for tumor microscopically (i.e. nodes attached to and removed with the primary tumor may be positive) }\end{array}$ \\
\hline Stage II A & $\begin{array}{l}\text { Localized tumor with incomplete gross excision; representative ipsilateral nonadherent lymph nodes negative for tumor } \\
\text { microscopically }\end{array}$ \\
\hline Stage II B & $\begin{array}{l}\text { Localized tumor with or without complete gross excision, with ipsilateral nonadherent lymph nodes positive for tumor. } \\
\text { Enlarged contralateral lymph nodes must be negative microscopically }\end{array}$ \\
\hline Stage III & $\begin{array}{l}\text { Unresectable unilateral tumor infiltrating across the midline, with or without regional lymph node involvement; or localized } \\
\text { unilateral tumor with contralateral regional lymph node involvement; or midline tumor with bilateral extension by infiltration } \\
\text { (unresectable) or by lymph node involvement. The midline is defined as the vertebral column. Tumors originating on one } \\
\text { side and crossing the midline must infiltrate to or beyond the opposite side of the vertebral column. }\end{array}$ \\
\hline Stage IV & $\begin{array}{l}\text { Any primary tumor with dissemination to distant lymph nodes, bone, bone marrow, liver, skin, and/or other organs, except } \\
\text { as defined for stage } 4 \mathrm{~S} \text {. }\end{array}$ \\
\hline Stage IV S & $\begin{array}{l}\text { Localized primary tumor, as defined for stage } 1,2 \mathrm{~A} \text {, or } 2 \mathrm{~B} \text {, with dissemination limited to skin, liver, and/or bone marrow } \\
\text { (limited to infants younger than } 18 \text { months). }{ }^{5} \text { Marrow involvement should be minimal (i.e. }<10 \% \text { of total nucleated cells } \\
\text { identified as malignant by bone biopsy or by bone marrow aspirate). More extensive bone marrow involvement would be } \\
\text { considered stage } 4 \text { disease. The results of the MIBG scan, if performed, should be negative for disease in the bone marrow. }\end{array}$ \\
\hline
\end{tabular}


- Biochemical diagnosis is based on elevated 24-hour urinary catecholamines/metanephrines, particularly dopamine and their metabolites. Normal catecholamine and metanephrine levels do not exclude neuroblastoma/ ganglioneuroblastoma.

- Only a minority of these patients will manifest clinical features of catecholamine excess.

- Alpha-blockade is advised for patients with noradrenaline-producing tumors.

- Meta-iodobenzylguanidine is very sensitive for localizing and assessing the extent of the disease.

- No specific protocols are available for treating adults with neuroblastoma/ganglioneuroblastoma; pediatric treatment guidelines are currently used.

- There is only one prior report of neuroblastoma occurring in pregnancy, as in this second case, treatment consisted of elective cesarean section prior to tumor resection. No apparent negative effects on the fetus were observed in either case.

- Treatment consists of surgical resection, more advanced disease requires chemo/radio/MIBG therapy. The prognosis for the adults with retroperitoneal neuroblastoma remains poor with current therapeutic strategies.

\section{REFERENCES}

1. Davis S, Rogers MA, Pendergrass TW. The incidence and epidemiologic characteristics of neuroblastoma in the United States. Am J Epidemiol 1987;126(6):1063-1074.

2. Esiashvili N, Goodman M, Ward K, et al. Neuroblastoma in adults: incidence and survival analysis based on SEER data. Paediatric blood and cancer 2007;49(1):41-46.

3. Schipper M, Van Duinen SG, Taphoon MJ, et al. Cerebral ganglioneuroblastoma of adult onset: two patients and review of literature. Clinical Neurology and Neurosurgery 2012;114(6):529-534.

4. Allan SG, Cornbleet MA, Carmichael J, et al. Adult neuroblastoma: report of three cases and review of the literature. Cancer 1986;57(12):2419-2421.

5. Aleshire SL, Glick AD, Gruz VE, et al. Neuroblastoma in adults. Pathologic findings and clinical outcomes. Arch Pathol Lab Med 1985;109(4):352-356.

6. Kilton L, Aschenbrener C, Burns CP. Ganglioneuroblastoma in Adults. Cancer 1976;37(2):974-983.

7. Isaacs $\mathrm{H}$, Medalie M, Politzer WM. Noradrenaline-secreting neuroblastomata. Br Med J 1959;1(5119):401-404.

8. Sandler M. Catecholamine-secreting tumours. Proc R Soc Med 1967;60(8):795-797.

9. Blijham GH, Barlogie B, Richman S, et al. Medulloblastoma and Neuroblastoma in adults. Neth J Med 1982;25(4):94-99.

10. Chaudhury A. Abdominal sympathoblastoma in adult with lung abscess. J Ind Medicine 1975;64(9):240-242.

11. Hale JE. A case of neuroblastoma in an adult with raised catecholamines. Br J Surg 1970;57(7):551-553.

12. Rowe PH, Oram JJ, Scott GW. Neuroblastoma in adults. Post Grad Med J 1979;55(646):579-580.

13. Franks LM, Bollen A, Seeger RC, et al. Neuroblastoma in adults and adolescents. An indolent course with poor survival. Cancer 1997;79(10):2028-2035.
14. Yapanoglu T, Ozbey I, Aksoy Y, et al. Neuroblastoma in Adulthood. Turk J Med Sci 2008;38(3):281-283.

15. Ohashi K, Sawada H, Kanaizumi T, et al. Adult neuroblastoma in pregnancy: report of a case. Surgery Today 1993;23(8):742-746.

16. Kawakami M, Koda M, Matsunaga N, et al. Adult type neuroblastoma originated in retroperitoneum beginning with obstructive jaundice. J Clin Imaging 2001;25(4):284-287.

17. Nzegwu M, Aghaji A. Neuroblastoma occurring in a 38-year-old Nigerian man: a rare finding. Rare Tumours 2009;1(1):42-43.

18. Lopez R, Karakousis C, Rao U. Treatment of adult neuroblastoma. Cancer 1980;45(5):840-844.

19. Dosik GM, Rodriguez V, Benjamin RS, et al. Neuroblastoma in the adult. Effective combination chemotherapy. Cancer 1978; 41(1):56-63.

20. Hasewaga T, Hirose T, Ayala AG, et al. Adult neuroblastoma of the retroperitoneum and abdomen: clinicopathologic distinction from primitive neuroectodermal tumor. Am J Surg Pathol 2001; 25(7):918-924.

21. Mackay B, Luna MA, Butler JJ. Adult neuroblastoma. Electron microscopic observations in 9 cases. Cancer 1976;37(3):1334-1351.

22. Prestidge BR, Donaldson SS. Treatment results among adults with childhood tumours: a 20 year experience. Int J Radiat Oncol Biol Phys 1989;17(3):507-514.

23. Baumgartner G, Gaeta J, Wajsman Z, et al. Neuroblastoma presenting as renal cell carcinoma in an adult. Urology 1975; 6(3):376-378.

24. Conte M, Parodi S, De Bernardi B, et al. Neuroblastoma in adolescents. Cancer 2006;106(6):1409-1417.

25. Yamanaka M, Saitoh F, Saitoh H, et al. Primary retroperitoneal ganglioneuroblastoma in an adult. Int J Urology 2001;8(3):130-132.

26. Koike K, Iihara M, Kanbe M, et al. Adult type ganglioneuroblastoma in the adrenal glan treated by a laparoscopic resection: report of case. Surgery Today 2003;33(10):785-790.

27. Nyaradi A, Kelle L, Klabuzai Z. High catecholamine-producing retroperitoneal ganglioneuroblastoma in adult. Orv Hetil 1971; 112(36):2162-2164.

28. Jalleh RP, Newman PL, Jenkins MT, et al. Primary renal ganglioneuroblastoma in adult. Post Grad Med J 1990;66(780):869-971.

29. Peycru T, Guiramand J, Tardat E, et al. Nodular ganglioneuroblastoma in adults. Canadian J Surgery 2009;52(4):E111-E113.

30. Kurt E, Filiz G, Vuruskan H, et al. Life-treatening complications of extremely rare tumour of the kidney: adult-type primary renal ganglioneuroblastoma. Sourth Med J 2007;100(8):852-853.

31. Sabharwal KK, Chauhan AL, Taterh A. Retroperitoneal ganglioneuroblastoma in an adult with itratumoural bleed. Ind $\mathrm{J}$ Radiolog Image 2006;16(2):193-196.

32. Mehta N, Tripathi RP, Popli MB, et al. Bilateral intra-abdominal ganglioneuroblastoma in adult. Br J Radiology 1997;70:96-98.

33. Mason GA, Hart-Mercer J, Millar EJ, et al. Adrenaline-secreting neuroblastoma in infant. Lancet 1957;273(6990):322-325.

34. Kaser H, Bettex M, V Studnitz W. Further observations on the determination of catecholamine metabolites in tumours of sympathetic nervous system. Arch Dis Childh 1964;39(204):168-171.

35. Voorhess M, Garderner LA. Studies of Catecholamine excretion by children with neural tumours. J Clin Endocrinol 1962;22: 126-133.

36. Sourkes T, Denton RL, Murphy GF, et al. The excretion of dixydrophenylanine, dopamine and dihydroxyphenylacetic acid in neuroblastoma. Pediatrics 1963;31:660-668.

37. Von Studnitz W, Kaser H, Sjoerdsma A. Spectrum of catechol amine biochemistry in patients with neuroblastoma. NEJM 1963; 269:232-235. 
38. Candito M, Thyss A, Albertini M, et al. Methylated catecholamine metabolites for diagnosis of neuroblastoma. Med Paed Oncology 1992;20(3):215-220.

39. Elbdeeb BB, Hammond Em, Worthington DJ, et al. Urinary catecholamines and their metabolites in management of neuroblastoma. Paed Haem Oncol 1988;5(3):229-237.

40. Marsden HB, Steward JL. Ewing's tumours and neuroblastomas. J Clin Path 1964;17(4):411-417.

41. Roberts NB, Dutton J, White MC, et al. An adrenaline-secreting ganglioneuroblastoma with elevated urinary HMMA but normal metanephrine secretion. Ann Clin Biochem 1992;29(pt 6):678-680.

42. Robinson R, Smith P, Whittakar SR, et al. Secretion of catecholamines in malignant paechromocytoma. BMJ 1964;1(5395):14221424.

43. Studnitz V, Kiser H, Sjoerdsma A. Spectrum of catecholamine biochemistry in patients with neuroblastoma. N Engl J Med 1963;269:232-235.

44. Sandler M, Calner DB, Karoum F, et al. Neuroblastoma. Biochemical studies, in recent results in cancer research. In: Bohuon, editor. Berlin; 1966. p. 55

45. Haberkern CM, Coles PG, Morray JP, et al. Intraoperative hypertension during surgical excision of neuroblastoma case report and review of 20 years experience. Anesth Analg 1992;75(5):854-858.

46. Jacobson AF, Deng H, Lombard J, et al. 123I-meta-iodobenzylguanidine scintigraphy for the detection of neuroblastoma and pheochromocytoma: results of a meta-analysis. J Clin Endocrinol Metabol 2010;95(6):2596-2606.

47. Brodeur GM, Pritchard J, Berthold J, et al. Revisions of the international criteria for neuroblastoma diagnosis, staging, and response to treatment. J Clin Oncol 1993;11(8):1466-1467.

48. Hiroshige K, Sonoda S, Fujita M, et al. Primary adrenal ganglioneuroblastoma in adult. Internal Medicine 1995;34(12): 1168-1173.

49. Brodeur JM, Hogarty MD, Mosse YP, et al. Neuroblastoma. Principles and practice of paediatric oncology. Philadelphia: Lippincott Williams and Wilkings; 2002. p. 895-937.

50. Rosen EM, Cassidy J, Frantz C, et al. Neuroblastoma. The joint centre for radiation therapy/dana-farber cancer institute/ children's hospital experience. J Clin Oncol 1984;2(7):719732.

51. Kretschmar C, Frantz CM, Rosen EM, et al. Improved survival in stage II and improved response rate in stage IV neuroblastoma. Proc Am Soc Clin Oncol 1984;2(7):799-803.

52. Kaye JA, Warhol MJ, Kretschmar C, et al. Neuroblastoma in adults-three case reports and review of the literature. Cancer 1986;58(5):1149-1157.

53. Matthay KK, Yanik G, Messina J. Phase II study on the effect of disease sites, age, and prior therapy on response to iodine131-metaiodobenzylguanidine therapy in refractory neuroblastoma. J Clin Oncol 2007;25(9):1054-1060. 